\title{
Generating a Novel Bispecific Nanobody to Enhance Antitumor Activity
}

\author{
Qiuhan Ge ${ }^{1}$ Tianyuan Sun ${ }^{1}$ Yanlin Bian ${ }^{2}$ Xiaodong Xiao ${ }^{3,4}$ Jianwei Zhu 2,3,4 \\ ${ }^{1}$ College of Pharmacy and Chemistry, Dali University, Yunnan, \\ People's Republic of China \\ 2 Engineering Research Center of Cell \& Therapeutic Antibody, \\ Ministry of Education, School of Pharmacy, Shanghai Jiao Tong \\ University, Shanghai, People's Republic of China \\ 3 Jecho Laboratories, Inc., Maryland, United States \\ Address for correspondence Jianwei Zhu, PhD, Engineering Research \\ Center of Cell \& Therapeutic Antibody, Ministry of Education, School \\ of Pharmacy, Shanghai Jiao Tong University, 800 Dongchuan Road, \\ Shanghai 200240, People's Republic of China \\ (e-mail: jianweiz@sjtu.edu.cn).
}

${ }^{4}$ Jecho Biopharmaceuticals Co., Ltd., Tianjin, People's Republic of China

\begin{abstract}
Keywords

- bispecific nanobody

- HER2

- VEGFR2

- colorectal cancer

Tumor cells express high levels of human epidermal growth factor receptor 2 (HER2) and vascular endothelial growth factor receptor 2 (VEGFR2), which are closely related to their proliferation and survival. Cancer treatments that target a single signaling pathway may result in immune pathway escape or drug resistance. Based on the correlation between the HER2 and VEGFR2 signaling pathways, we speculated that targeting the two pathways simultaneously may produce a synergistic effect and avoid occurrence of drug resistance, resulting in improved efficacy. Anti-VEGFR2 nanobody 3VGR19-3 and anti-HER2 nanobody 2D3 were combined to construct a bispecific nanobody (Bi-Nb). They can recognize both HER2 and VEGFR2 (both highly expressed in HT-29 cells) to simultaneously block the two signaling pathways. We verified the affinity of the Bi-Nb to its targets using the surface plasmon resonance technology, and test its effects to inhibit tumor cell growth and promote cell apoptosis in vitro by the Cell Counting Kit- 8 assay and apoptosis assay. In summary, we have successfully constructed a Bi-Nb, and verified its tumor-suppressing effects in vitro. Compared with a single monospecific nanobody, our Bi-Nb showed superior antitumor effect, which provides a new perspective for treatment of tumors with high HER2 and VEGFR2 expression.
\end{abstract}

\section{Introduction}

Colorectal cancer (CRC) is a common malignancy of the digestive tract, which accounts for a significant portion of cancerrelated deaths around the world. ${ }^{1}$ While human epidermal growth factor receptor 2 (HER2), a member of the ErbB family, is expressed on the surface of normal endothelial cells, it is often overexpressed on tumor cells, ${ }^{2}$ causing tumors with elevated HER2 expression highly invasive. Vascular endothelial growth factor receptor 2 (VEGFR2) is involved in the regulation of angiogenesis, therefore, inhibiting VEGFR2 activity, and the downstream signaling pathway has become an important approach in cancer treatment. ${ }^{3-5}$ Overexpression of HER2 can upregulate VEGF through the PI3K signaling pathway, so blocking HER2 significantly decreases VEGF expression and helps killing the tumor cells., ${ }^{3,6}$ In addition, based on the synergetic effect and cross-talk between the signaling pathways, targeting both receptors simultaneously may more effectively inhibit tumor cell proliferation and promote apoptosis. To date, chemotherapy has been widely used for clinical cancer treatment, but long-term use of these agents will lead to drug resistance and bone marrow suppression. ${ }^{7}$ To reduce the side effects on cancer patients, approaches with targeted drug delivery represent a better choice. Novel genetic mechanisms in gene expression and targeting have been reported in the last several years. ${ }^{8}$ Targeted drugs are delivered specifically to the site of tumor, so they can be concentrated there and more effectively kill the tumor cells, and meanwhile, exert less 
damage to normal tissue. ${ }^{9-12}$ Antibodies and bispecific antibodies have become the most important part of targeted tumor therapy. ${ }^{13-16}$ Bispecific Antibody by Protein TransSplicing (BAPTS) has been reported to synthesize BsAbs with natural human immunoglobulin $G(\operatorname{IgG})$ structure and no chain mispairing. ${ }^{17,18}$ Nanobodies, such as camel-derived antibodies, that naturally lack the light chains and contain only the variable region of the heavy chain are the smallest antigenbinding fragments. ${ }^{19}$ Compared with traditional monoclonal antibodies, nanobodies have the advantages of small size, high solubility, high stability, high affinity to their targets, low immunogenicity, and excellent tissue penetration..$^{20,21}$ Based on the characteristics of nanobodies and the special structure of VHH of the natural heavy-chain antibody, ${ }^{21,22}$ previously our laboratory has shown that nanobodies combined with immunotoxin improve antitumor activity. ${ }^{23}$ In this study, we developed a bispecific nanobody (Bi-Nb) using anti-HER2 and anti-VEGFR2 nanobodies. This Bi-Nb targets simultaneously HER2 and VEGFR2 in tumor cells overexpressing these two receptors, ${ }^{24,25}$ which holds greater potential in medical research and clinical treatment of cancer.

\section{Materials and Methods}

\section{Cell Lines and Reagents}

The HT-29 cells used in this study were preserved in our laboratory and cultured on the McCoy's 5A medium (McCoy's 5A; Gibco, United States), supplemented with $10 \%(\mathrm{v} / \mathrm{v})$ fetal bovine serum (FBS; Gibco, USA) and 1\% (v/v) penicillin/streptomycin $(\mathrm{P} / \mathrm{S}$; Hyclone, United States). All the cultures were kept in a plastic flask and incubated at $37^{\circ} \mathrm{C}$ in $5 \% \mathrm{CO}_{2}$. The Cell Counting Kit-8 (CCK-8) kit was purchased from Dongren Chemical Technology (Shanghai) Co., Ltd., BCA kit was purchased from Shanghai Biyuntian Biotechnology Co., Ltd., and Ni-NTA gel from Nanjing Kingsley Co., Ltd. Yeast extract, tryptone, and peptone were purchased from OXOID Co., Ltd. Flow cytometry used in this study was CytoFlex from Beckman. VEGFR2 antibody (2C6) was purchased from Novus Biologicals (United States). VEGF165 and PE-conjugated VEGFR2 antibody were purchased from Sino Biological (Beijing, China). FITCconjugated rat anti-mouse IgG1 secondary antibody and FITCconjugated $6 \times$ His tag $\mathrm{mAb}$ (AD1.1.10) were purchased from Thermo Fisher (United States).

\section{Construction and Expression of Bispecific Nanobody}

The 2D3 and Bi-Nbs were synthesized by Synbio Technologies (Soochow, China). The sequence information of the antiHER2 2D3 nanobody was obtained from Ablynx. ${ }^{25}$ The sequence information of the anti-VEGFR2 3VGR19-3 nanobody was provided by our laboratory. Then they were cloned into plasmid pET-22b(+) between restriction sites NcoI and Xhol. Primers used were T7 and T7 terminals. The above three nanobodies were confirmed by sequencing. The constructed plasmid was transformed into Escherichia coli strain BL21 (DE3). Inoculum 1\% (v/v) was inoculated to $1 \mathrm{~L}$ culture, and $1 \mathrm{mmol} / \mathrm{L}$ IPTG inducer was added when the $\mathrm{OD}_{600}$ value of the bacterial liquid reached 0.5 to 0.6 . After centrifugation $(12,000 \mathrm{r} / \mathrm{min}, 10$ minutes $)$, periplasmic protein was extracted by osmotic shock and purified by an affinity metal chromatography column (nickel column), then analyzed by $15 \%$ SDS-PAGE and Western blotting with anti-His tag mouse antibody.

\section{Surface Plasmon Resonance Kinetics Measurements}

Affinity constants for the binding between $\mathrm{Bi}-\mathrm{Nb}$ and VEGFR2 or HER2 were determined by surface plasmon resonance (SPR) analysis using the Biacore T200 system. The VEGFR2 or HER2 was immobilized on the CM-5 sensor chip. There are four channels on the CM- 5 sensor chip, two coupling VEGFR2 or HER2 interaction detection channels and two nonfixed VEGFR2 or HER2 as blank reference channels. At a flow rate of $30 \mu \mathrm{L} / \mathrm{min}$, HBS solution was used as the working solution, and the gradient concentrations of nanobodies were injected respectively, each concentration was detected twice. All Biacore kinetics experimental data were obtained using the Biacore T200 evaluation software to estimate the association rate constant $\left(k_{\mathrm{a}}\right)$ and dissociation rate constant $\left(k_{\mathrm{d}}\right)$.

\section{Flow Cytometry Analysis}

After washing three times with phosphate buffered saline (PBS)-2\% FBS, $2 \times 105$ HT-29 cells were incubated at $4^{\circ} \mathrm{C}$ for 30 minutes with $1 \mathrm{mg} / \mathrm{mL}$ nanobodies (2D3, 3VGR19-3, and $\mathrm{Bi}-\mathrm{Nb}$ ) in PBS-2\% FBS. After three times washing with PBS-1\% BSA $(w / v)$, cells were incubated with anti-His-tag FITC antibody for 30 minutes on ice. Excess fluorescein-labeled antibody was removed by washing with PBS-2\% FBS. Then, the cells were fully suspended with $400 \mu \mathrm{L}$ PBS and analyzed by flow cytometry.

\section{CCK-8 Cell Proliferation Assay}

The CCK-8 assay kit (Sigma-Aldrich) was used to detect the effect of nanobodies on the cell viability of HT-29 cells. Briefly, the starved cells were seeded into separate 96-well plates at 3,000 cells/well and cultured overnight at $37^{\circ} \mathrm{C}$. At the end of incubation, $10 \mu \mathrm{L}$ CCK-8 was added to each well, and the cells were counted by absorbance measurements at a wavelength of $450 \mathrm{~nm}$. The cell survival rate (\%) of the target cells was calculated using the following formula: $\left[\left(A_{\mathrm{s}}-A_{\mathrm{b}}\right) /\right.$ $\left.\left(A_{0}-A_{\mathrm{b}}\right)\right] \times 100 \%$, where $A_{\mathrm{s}}, A_{\mathrm{b}}$, and $A_{0}$ are the absorbent values of the sample group, respectively. The $\mathrm{IC}_{50}$ values were then calculated by curve fitting using the GraphPad Prism software (San Diego, United States).

\section{Apoptosis Assay}

Apoptosis assay was performed with an Annexin V-FITC/ propidium iodide (PI) kit (Lianke, Hangzhou, China). A total of $5 \times 10^{5}$ HT29 cells were seeded in a 6-well plate. After adding the apoptosis-inducing agent, the cells were incubated at $37^{\circ} \mathrm{C}$ for 48 hours, then stained with PI and Annexin V-FITC for apoptosis assay on a flow cytometer. Anenxin V-FITC/PI double staining can detect cell apoptosis and differentiate into early apoptotic cells (annexin $\mathrm{V}^{+} / \mathrm{PI}^{-}$), late apoptotic cells (annexin $\mathrm{V}^{+} / \mathrm{PI}^{+}$) and necrotic cells (annexin $\mathrm{V}^{-} / \mathrm{PI}^{+}$) Apoptosis. The percentage of apoptotic cells was calculated as the total percentage of early apoptotic cells and late apoptotic cells. 


\section{Results}

Construction, Expression, and Purification of the Bi-Nb The construction of the mono-Nb and Bi-Nb is illustrated in -Fig. 1 and - Table 1. We designed forward and reverse primers for plasmid construction and subcloned them into the vector pET-22b $(+)$. The target gene sequence was confirmed by DNA sequencing. The sequence information of 2D3, 3VGR19-3, and Bi-Nb was confirmed by sequencing. The genes of interest were expressed in E. coli; six anti-His tags were added and purified with a nickel column. 2D3 and 3VGR19-3 were present as a single band of $14 \mathrm{kDa}$ ( - Fig. 1A). Bi-Nbs were constructed by linking two nanobodies, antiVEGFR2 and anti-HER2, with a (G4S)3 linker. The recombinant protein was present as a single band of $\sim 28 \mathrm{kDa}$ (-Fig. 1B). The final yield was $5 \mathrm{mg} / \mathrm{L}$ for 2D3, $1 \mathrm{mg} / \mathrm{L}$ for 3VGR19-3, and $4 \mathrm{mg} / \mathrm{L}$ for the Bi-Nb.

\section{Bi-Nb Binding to VEGFR2/HER2 Analyzed by SPR}

Evaluation of the binding of antigens VEGFR2 or HER2 with Bi-Nb was performed by SPR analysis, as shown in - Fig. 2. The SPR technology was used to detect the binding affinity of 2D3 to HER2 (- Fig. 2A) and 3VGR19-3 to VEGFR2 (- Fig. 2B). The detailed kinetic parameters are listed in - Table 2. A hyperbolic curve was formed by the combination of the two antigens with the Bi-Nb (-Fig. 2E). Based on the results of the affinity constant, we can find that the ability of $\mathrm{Bi}-\mathrm{Nb}$ to recognize antigens is weaker than that of single-target nanobody. We consider that the high molecular weight of Bi-Nbs limits its ability to bind to antigens.

\section{Affinity Analysis of the Nanobodies to HT-29 Cell Surface Antigen}

The ability of individual nanobody and $\mathrm{Bi}-\mathrm{Nb}$ for the recognition of receptor on the HT29 cell surface was analyzed by flow cytometry. The results showed that 2D3, 3VGR19-3, and Bi-Nb bound to HER2 or VEGFR2 in HT-29 cells (-Fig. 3). 2D3,
3VGR19-3, and Bi-Nb had certain affinity to antigen in HT-29 cells; therefore, we chose to use HT29 cells to detect the efficacy of antibodies for against tumors.

\section{Bi-Nb Inhibits the Proliferation of HT29 Cells}

The biological activities of the nanobodies were tested by the CCK-8 kit assay, such as inhibition of cell proliferation, migration, and induction of cell apoptosis were verified. Inhibition of cell proliferation may indicate a possibility for cancer treatment. We measured cell viability in HT29 cells treated with different concentrations of nanobody or Bi-Nb using CCK-8 assays. The results are shown in - Fig. 4. The $\mathrm{IC}_{50}$ value of $2 \mathrm{D} 3$ was $168.2 \mathrm{nmol} / \mathrm{L}$, that of $3 \mathrm{VGR} 19-3$ was $584.4 \mathrm{nmol} / \mathrm{L}$, while that of the Bi-Nb was $101.2 \mathrm{nmol} / \mathrm{L}$.

\section{Bi-Nb Induces Apoptosis}

As shown in - Fig. 5, HT29 cells were treated with $200 \mathrm{~nm}$ nanobody, followed by apoptosis assay with the FITC-Annexin $\mathrm{V} / \mathrm{PI}$ kit to determine cytotoxicity of the nanobodies to the cells, and then with annexin V-FITC and PI to distinguish populations of early apoptotic (annexin $\mathrm{V}^{+} / \mathrm{PI}^{-}$), late apoptotic (annexin $\mathrm{V}^{+} / \mathrm{PI}^{+}$) cells. The results showed that the Bi-Nb was more effective in inducing apoptosis of the tumor cells.

\section{Discussion and Conclusions}

Studies on CRC risk have identified potential factors associated with the disease. ${ }^{26-30}$ A close correlation has been detected between HER2 overexpression and elevated VEGF in several human tumors, especially breast cancer. ${ }^{31,32}$ Simultaneously blocking HER2 and VEGFR2 signaling pathways with the Bi-Nb may overcome the drug resistance commonly seen after treatments targeting only one of them and produce synergistic antitumor effects, which would be of great clinical significance. $^{33-35}$ We chose to use the (G4S)3 linker to bind the two nanobodies together for constructing the novel Bi-Nb (-Fig. 1A). The results showed that (-Fig. 1B) the expression

$$
\text { Coding DNA Expression protein }
$$

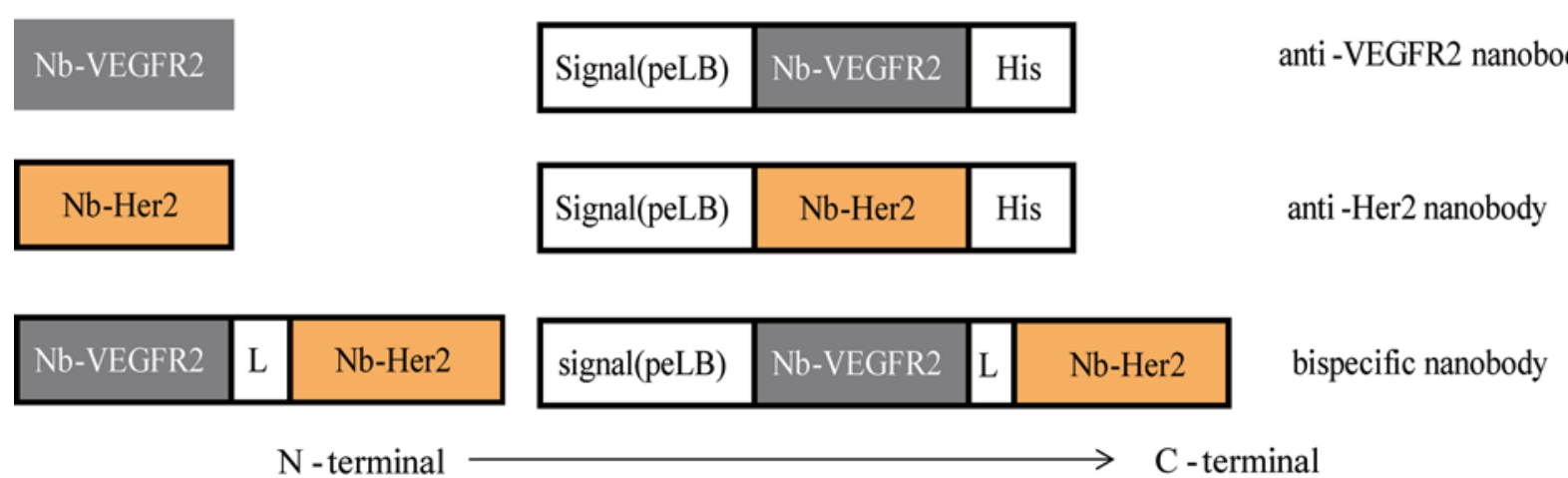

Signal(peLB):Signal peptide, His:histidine Label, L:linker

Fig.1 (A) Basic structure of anti-HER2 nanobody, anti-VEGFR2 nanobody, and bispecific nanobody. (B) Coomassie-stained SDS-PAGE and Western blotting analysis of the purified Bi-Nb and the Nbs. Expression and purification of 2D3 (1,2). Expression and purification of 3VGR19-3 (3, 4). Expression and purification of bispecific nanobody $(5,6)$. L is (G4S)3 (GGCGGCGGTGGTAGCGGCGGCGGCGGTTCCGGCGGTGGTGGTTCT) linker, $\mathrm{M}$ is size standards. PSP, periplasmic space protein; FT, flow through by IMAC; E20-E500, elution samples with 20-500 mmol/L imidazole. 

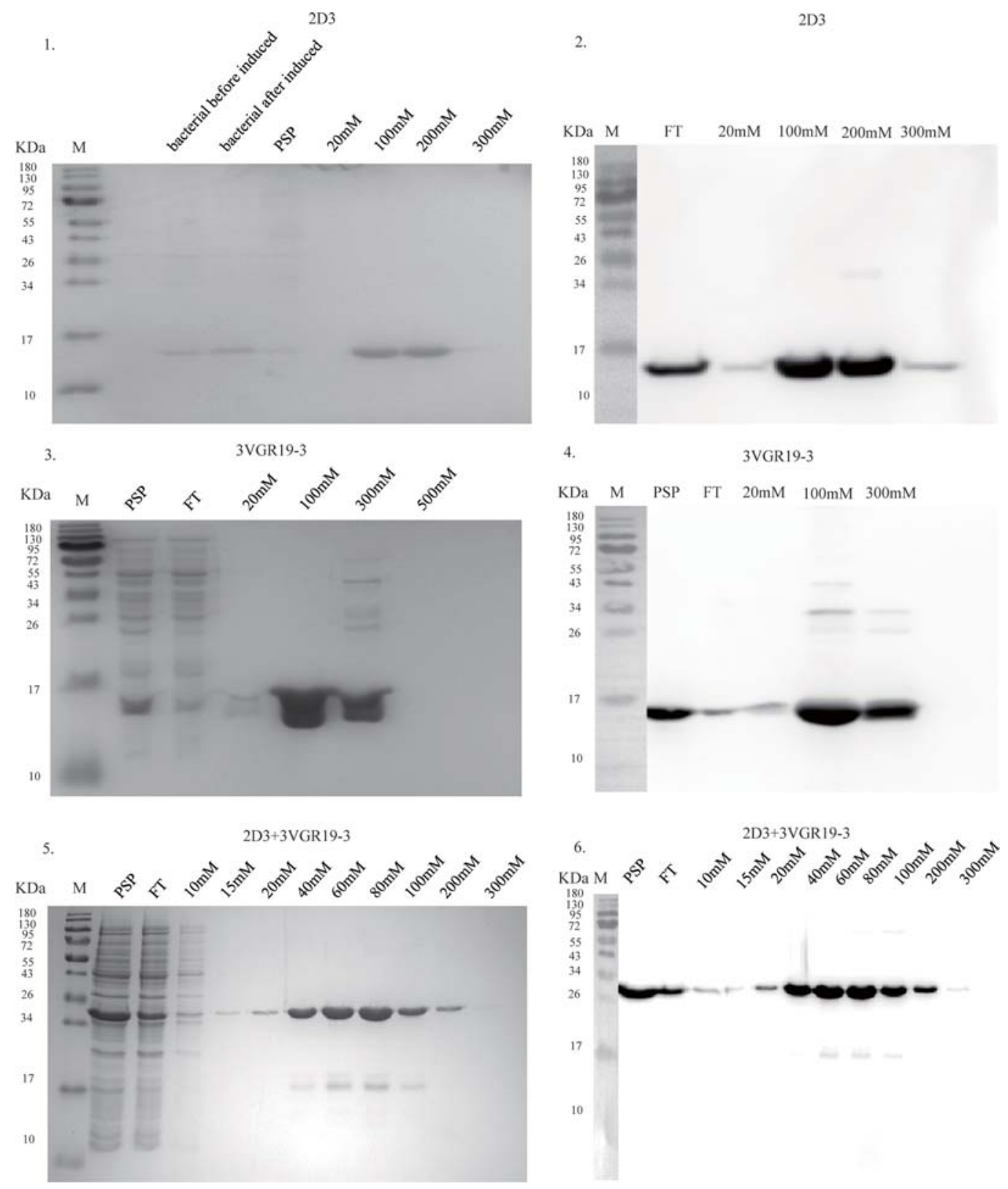

Fig.1 (Continued)

Table 1 The Primer Sequence applied for constructing nanobodies 2D3 and 3VGR19-3a

\begin{tabular}{|c|c|c|}
\hline Name & \multicolumn{2}{|l|}{ Primer sequence } \\
\hline \multirow[t]{2}{*}{$2 \mathrm{D} 3$} & Primer F-2D3 & ATGCGAATTCGAAGTTCAGCTGGTTGAA \\
\hline & Primer R-2D3 & ATGCCTCGAGGCTGCTCACGGTAACTTG \\
\hline \multirow[t]{2}{*}{ 3VGR19-3 } & Primer F-3VGR19-3 & ATGCGAATTCGAAGTTCAGCTGCAGGAA \\
\hline & Primer R-3VGR19-3 & ATGCCTCGAG AGAAGAAACGGTAACCTG \\
\hline \multirow[t]{4}{*}{3 VGR19-3 + 2D3 } & 3VGR19-3-F' & ATGCGAATTCGAAGTTCAGCTGCAGGAA \\
\hline & 3VGR19-3-R' & $\begin{array}{l}\text { ATGCCTCGAGAGAACCACCACCGCCGGAACCGCCGCCGCCGCTACCACCGCCGCC } \\
\text { AGAAGAAACGGTAACCTG }\end{array}$ \\
\hline & $2 \mathrm{D} 3-\mathrm{R}^{\prime}$ & RATGCCTCGAGGCTGCTCACGGTAACTTG \\
\hline & $2 \mathrm{D} 3-\mathrm{F}^{\prime}$ & $\begin{array}{l}\text { TGCGAATTCGGCGGCGGTGGTAGCGGCGGCGGCGGTTCCGGCGGTGGTGGTTCT } \\
\text { GAAGTTCAGCTGGTTGAA }\end{array}$ \\
\hline
\end{tabular}

aThe detailed primer sequence is shown in - Table 1. We amplified the target fragment by PCR on the primer sequence, then cloned into plasmid pET-22b(+) between restriction sites Ncol and Xhol. 

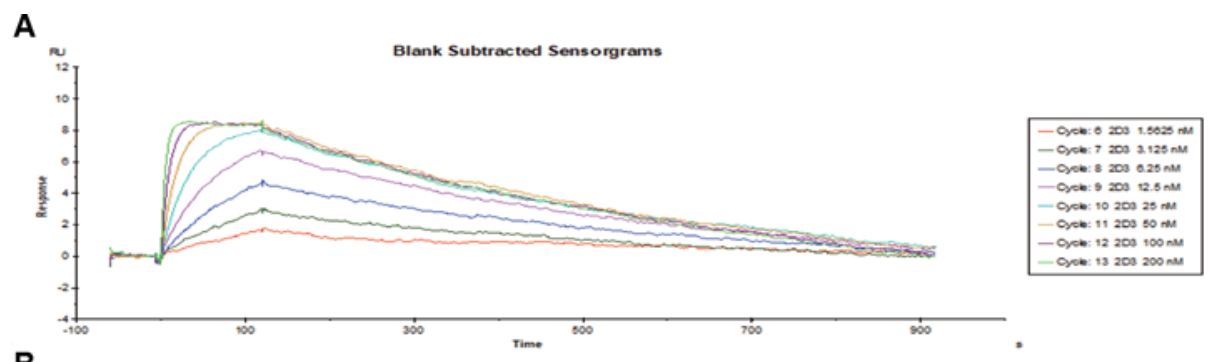

B

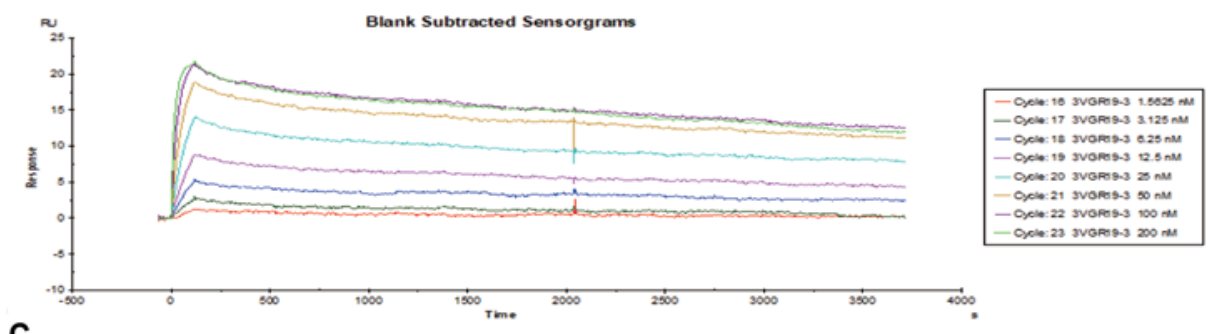

C
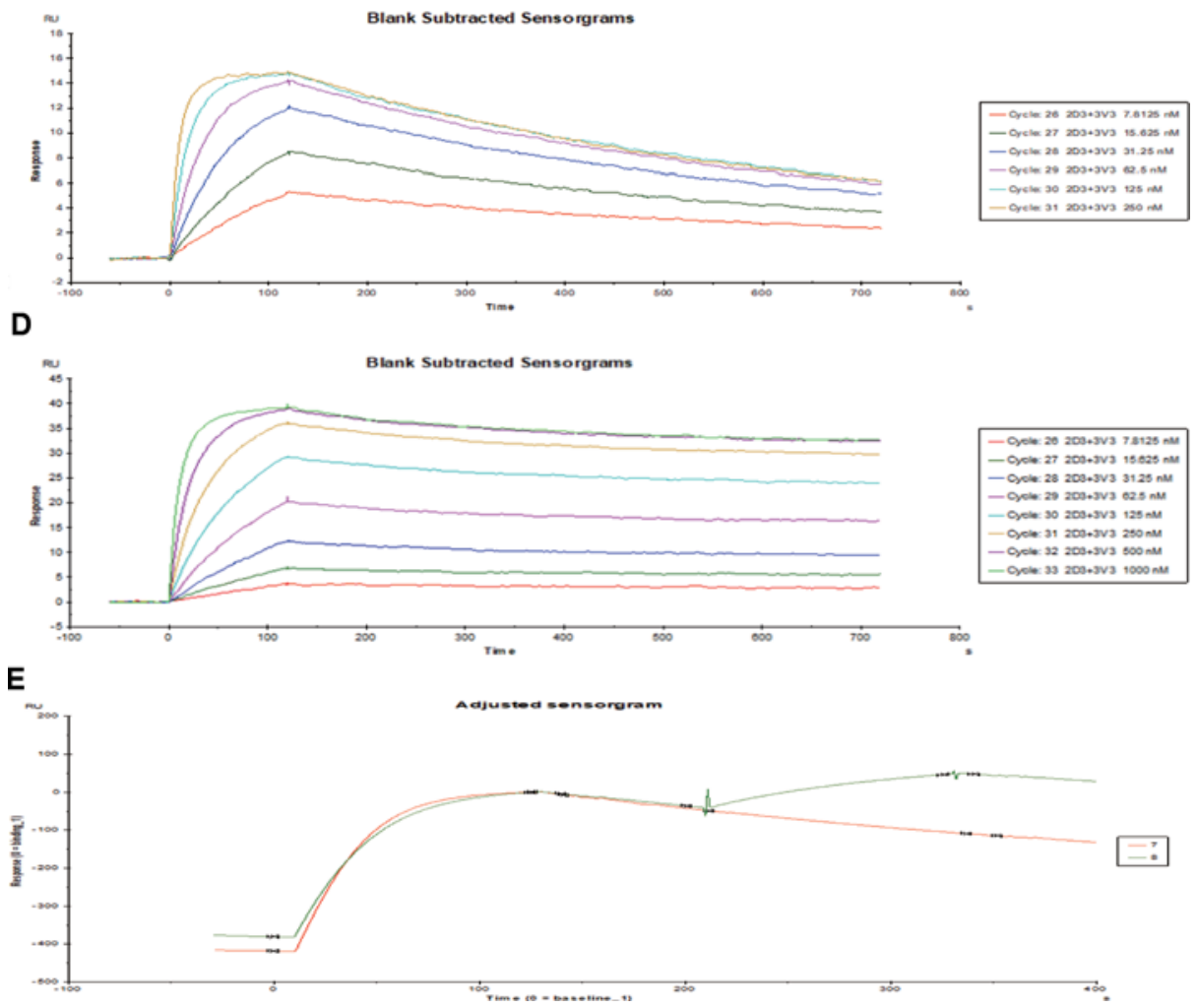

Fig. 2 Identification of affinity between nanobody and antigen by Biacore. (A) Determination of affinity between 2D3 and HER2. (B) Determination of affinity between 3VGR19-3 and VEGFR2. (C) Identification of affinity of bispecific nanobody to HER2. (D) Identification of affinity of bispecific nanobody to VEGFR2. (A-D) The curves of different colors represent different concentrations of nanobodies. (E) Hyperbolic binding of two antigens to bispecific nanobodies. The red curve represents the PBS group and the green curve represents the bispecific nanobody group. And the two peaks show that the bispecific nanobody first binds to VEGFR2, and then binds to HER2 at 200 seconds.

Table 2 Affinity constants of nanobodies to HER2 or VEGFR2 by Biacore analysis ${ }^{\mathrm{a}}$

\begin{tabular}{|l|l|l|l|l|}
\hline Nbs & Targets & $K_{\mathrm{a}} / \mathrm{mol}^{-1} \mathrm{~L} \mathrm{~s}^{-1}$ & $K_{\mathrm{d}} / \mathrm{s}^{-1}$ & $K_{\mathrm{D}} / \mathrm{mol} \mathrm{L}^{-1}$ \\
\hline 2D3 & HER2 & $1.342 \mathrm{E}^{+6}$ & 0.002768 & $2.063 \mathrm{E}^{-9}$ \\
\hline 3VGR19-3 & VEGFR2 & $4.388 \mathrm{E}^{+5}$ & $1.505 \mathrm{E}^{-4}$ & $3.430 \mathrm{E}^{-10}$ \\
\hline Bi-Nb & HER2 & $5.565 \mathrm{E}^{+5}$ & 0.001579 & $2.838 \mathrm{E}^{-9}$ \\
\hline Bi-Nb & VEGFR2 & $9.509 \mathrm{E}^{+4}$ & $2.978 \mathrm{E}^{-4}$ & $3.132 \mathrm{E}^{-9}$ \\
\hline
\end{tabular}

${ }^{\mathrm{a}}$ The association and dissociation constants $\left(K_{\mathrm{a}}, K_{\mathrm{d}}\right)$ were calculated using the Biacore T200 evaluation software. $K_{\mathrm{D}}$ was calculated from the quotient of $K_{\mathrm{d}} / K_{\mathrm{a}}$. 

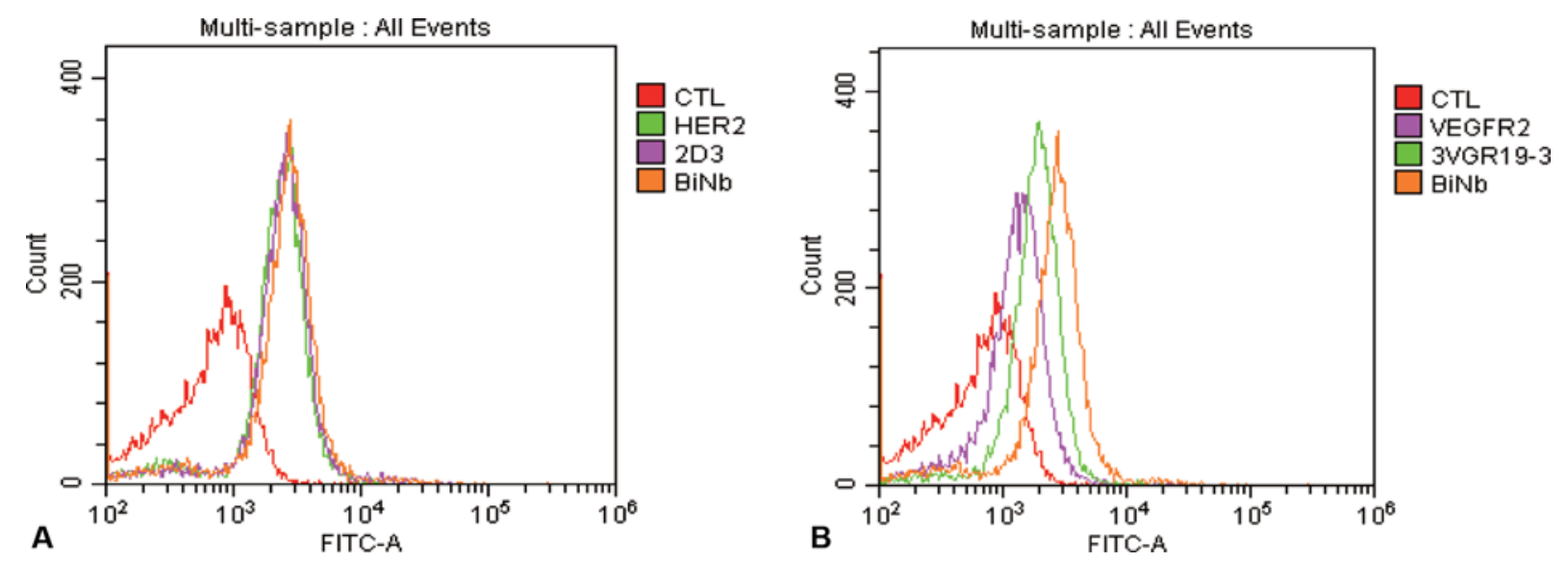

Fig. 3 Flow cytometry analysis results showing that bispecific nanobody binds to VEGFR2 and HER2 co-expressing HT-29 cells. In the process of detection, positive control HER2 (A) and positive control VEGFR2 (B) were found respectively.

level of Bi-Nb was the same as that of single nanobody and could be effectively secreted in periplasmic protein. The transient gene expression technology platform has been widely used in a variety of therapeutic proteins and monoclonal antibodies. ${ }^{36-38}$ Compared with the traditional Bi-Nbs, this Bi-Nb not only has a high yield, but also is easy to be purified. We consider that the high yield of the Bi-Nb may be due to its small molecular weight, high solubility, and the high expression of target protein in the prokaryotic system. ${ }^{39}$ In addition, in the process of purification, the proteins in the periplasmic space were used as the mother liquor for gradient elution, but there were fewer miscellaneous proteins in the cell periplasmic space, so it was easier to purify the target protein. The results of SPR and fluorescence-activated cell sorting showed that the Bi-Nb could target HER2 and

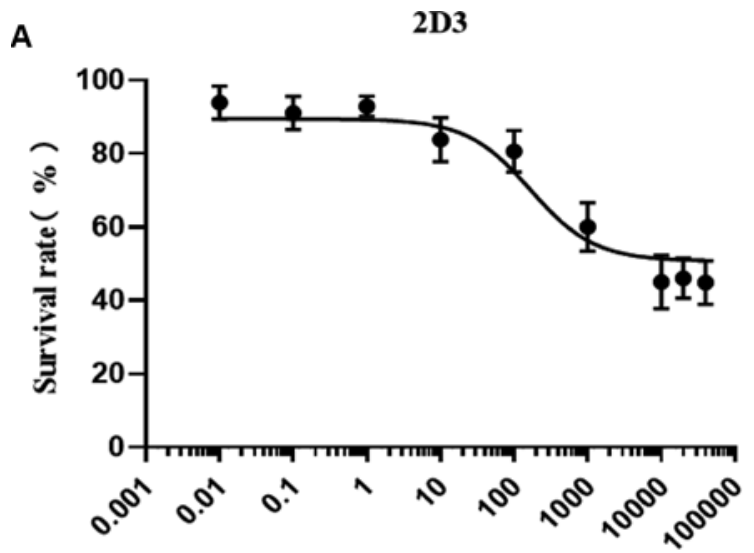

Concentration(nm)
B

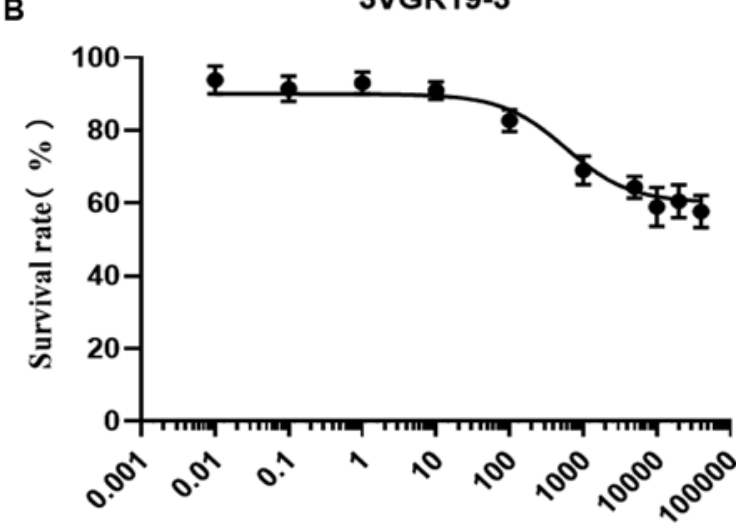

C

2D3+3VGR19-3

Concentration(nm)

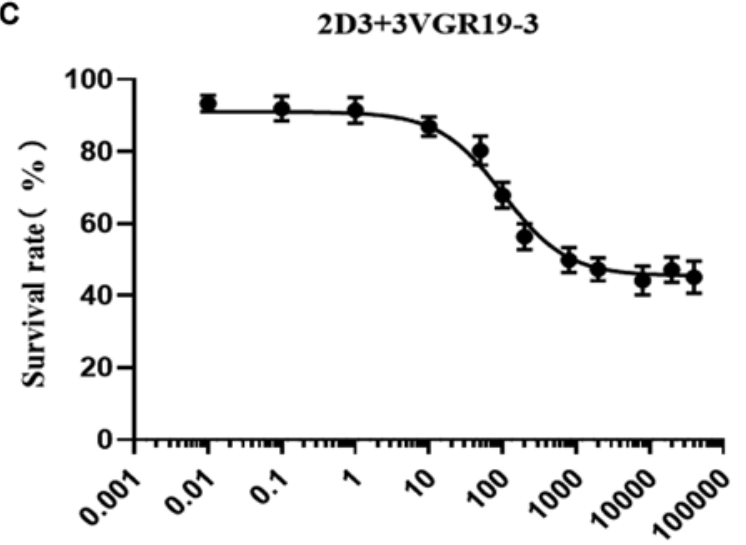

Concentration(nm)

Fig. 4 CCK-8 kit showing that bispecific nanobody inhibited HT-29 cell growth nanobody or Bi-Nb inhibited the proliferation of HT29 in a dosedependent manner. A CCK-8 assay was performed on HT29 $(3 \times 103$ cells/well). The IC 50 values were calculated by curve fitting using the GraphPad Prism software (values represented as means \pm SD, $n=3$ ). 
A

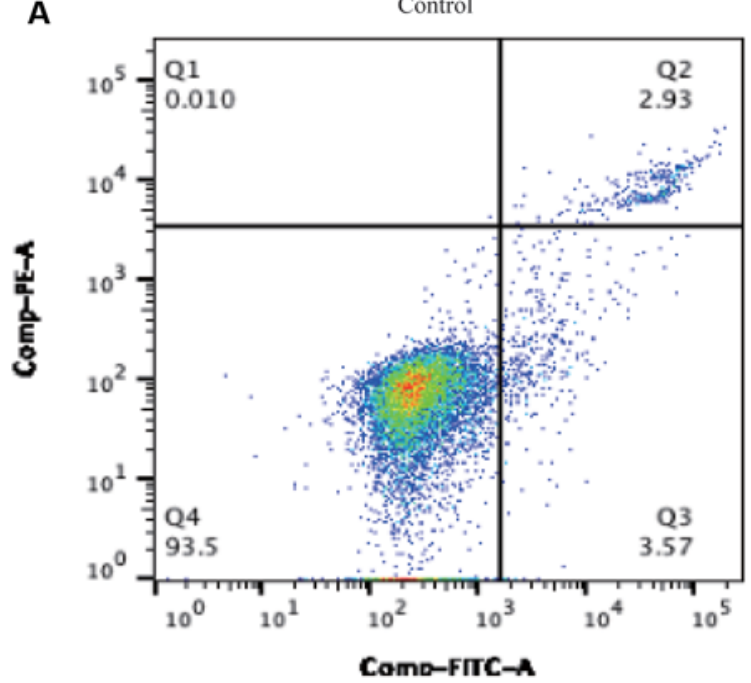

3VGR19-3

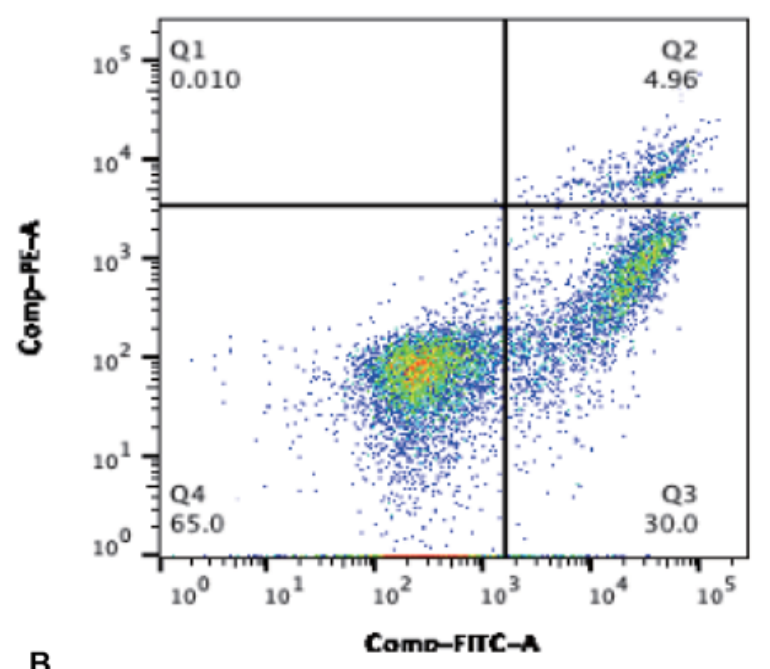

B

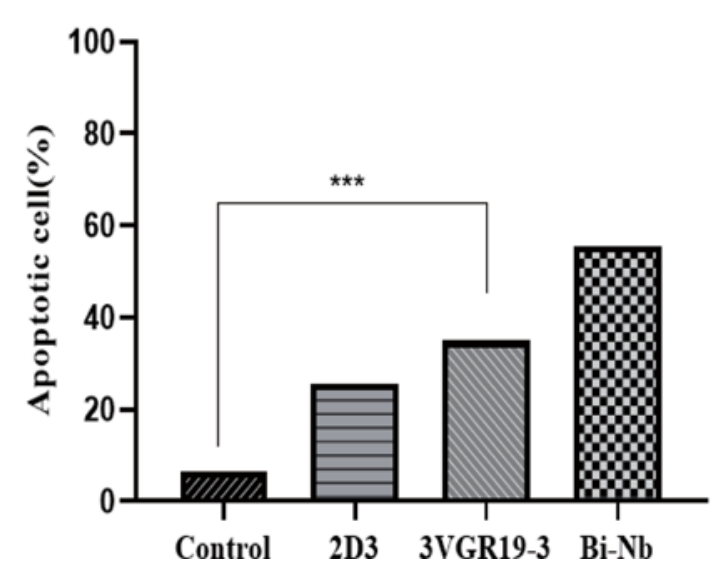

2D3

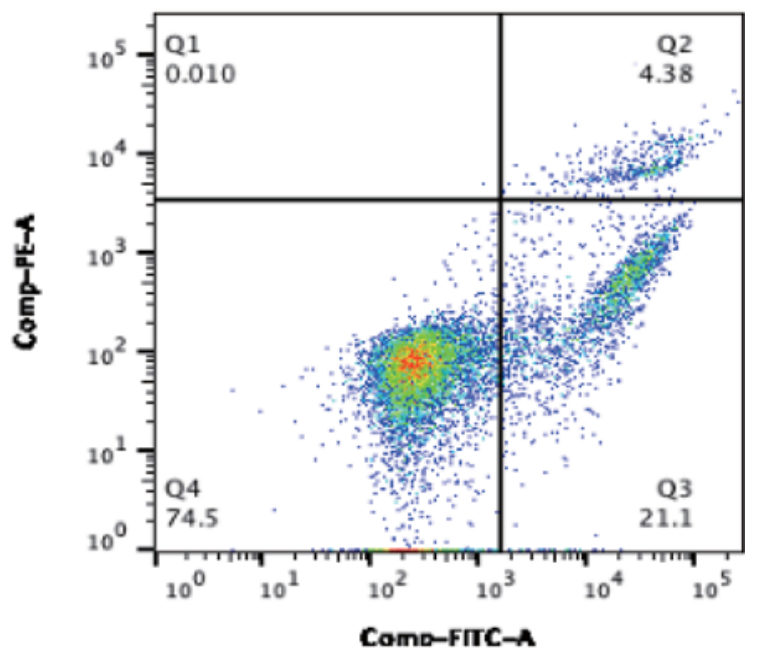

$\mathrm{Bi}-\mathrm{Nb}$

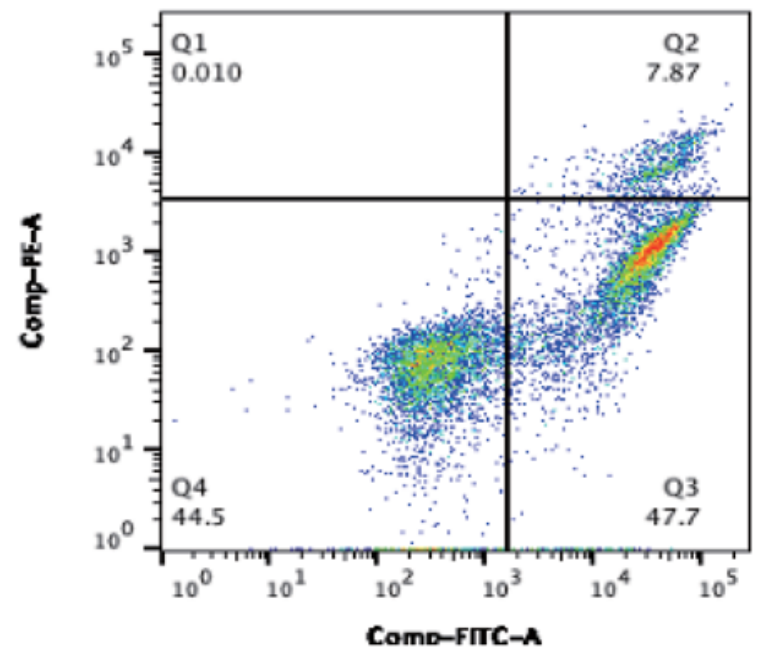

Fig. 5 After treatment specified for each group, HT29 cells exposed to $200 \mathrm{~nm}$ concentrations of nanobodies were stained with Annexin V-FITC and PI $(p<0.05)$. 2D3 or 3VGR 19-3 could decrease the percentage of proliferating HT29 cells by $\sim 30 \%$, from $93.5 \%$ to $74.5 \%$ or $65.0 \%$. Treatment with Bi-Nb markedly increased apoptosis in HT29 cells from 6.5\% (early apoptosis 3.57\% plus late apoptosis $2.93 \%$ ) to $\sim 55 \%(p<0.05$ ). Q1UL, necrotic; Q2UR, late apoptotic; Q3LR, early apoptotic; Q4LL, live.

VEGFR2. Next, biological activities or anticancer activities in vitro were evaluated by cell proliferation assay and apoptosis assay. The Bi-Nb inhibited HT29 cell proliferation in a dosedependent manner, with an $\mathrm{IC}_{50}$ of $\sim 100 \mathrm{nmol} / \mathrm{L}$ (-Fig. 4).
Treatment with Bi-Nb markedly increased apoptosis in HT29 cells from 6.5 to $55 \%$ ( - Fig. 5A). These observations collectively suggested that the $\mathrm{Bi}-\mathrm{Nb}$ demonstrated biological function of inhibition of cell proliferation, migration, and apoptosis in vitro. 
As the Bi-Nb has been successfully constructed and its efficacy against tumor cells has been preliminarily validated, the next stage of the study seeks to evaluate its efficacy in animal models. Generally, the Bi-Nb still has some limitations in clinical application. It is believed that blocking neovascularization would effectively inhibit tumor growth, but once the drug is withdrawn, relevant signaling pathways would be activated again, and the tumor would resume vascularization and continue growing. ${ }^{40}$ As seen in renal cell carcinoma treated with bevacizumab alone, tumors grow rapidly during the intervals of treatment. To address this problem, we shall further combine toxins with nanobodies into immunotoxins, or with other antitumor drugs. This study provides a new perspective for clinical treatment of tumor.

\section{Conflicts of Interest}

The authors declare no conflict of interest.

\section{Acknowledgments}

This project was supported by the National Natural Science Foundation of China (grant number: 81773621). Great thanks to Jianrong Xu (School of Medicine, Shanghai Jiao Tong University, Shanghai, China) for her help with SPR technology. This study does not contain experiments using animal and human subjects.

\section{References}

1 Gupta N, Kupfer SS, Davis AM. Colorectal cancer screening. JAMA 2019;321(20):2022-2023

2 Agus DB, Bunn PA Jr, Franklin W, Garcia M, Ozols RF. HER-2/neu as a therapeutic target in non-small cell lung cancer, prostate cancer, and ovarian cancer. Semin Oncol 2000;27(06, Suppl 11):53-63, discussion 92-100

3 Smith NR, Baker D, James NH, et al. Vascular endothelial growth factor receptors VEGFR-2 and VEGFR-3 are localized primarily to the vasculature in human primary solid cancers. Clin Cancer Res 2010;16(14):3548-3561

4 Larsen AK, Ouaret D, El Ouadrani K, Petitprez A. Targeting EGFR and $\operatorname{VEGF}(\mathrm{R})$ pathway cross-talk in tumor survival and angiogenesis. Pharmacol Ther 2011;131(01):80-90

5 Mitran B, Güler R, Roche FP, et al. Radionuclide imaging of VEGFR2 in glioma vasculature using biparatopic affibody conjugate: proof-ofprinciple in a murine model. Theranostics 2018;8(16):4462-4476

6 Nussenbaum F, Herman IM. Tumor angiogenesis: insights and innovations. J Oncol 2010;2010:132641

7 Zhou Y, Zhen M, Guan M, et al. Amino acid modified [70] fullerene derivatives with high radical scavenging activity as promising bodyguards for chemotherapy protection. Sci Rep 2018;8(01): 16573

8 Zhu J. Mammalian cell protein expression for biopharmaceutical production. Biotechnol Adv 2012;30(05):1158-1170

9 Movahedi MM, Mehdizadeh A, Koosha F, et al. Investigating the photo-thermo-radiosensitization effects of folate-conjugated gold nanorods on KB nasopharyngeal carcinoma cells. Photodiagn Photodyn Ther 2018;24:324-331

10 Roskoski R Jr. Small molecule inhibitors targeting the EGFR/ErbB family of protein-tyrosine kinases in human cancers. Pharmacol Res 2019;139:395-411

11 Wang X, Ouyang X, Chen J, Hu Y, Sun X, Yu Z. Nanoparticulate photosensitizer decorated with hyaluronic acid for photodynamic/ photothermal cancer targeting therapy. Nanomedicine (Lond) 2019; 14(02):151-167
12 Warda W, Larosa F, Neto Da Rocha M, et al. CML hematopoietic stem cells expressing IL1RAP can be targeted by chimeric antigen receptor-engineered T cells. Cancer Res 2019;79(03):663-675

13 Khalil DN, Smith EL, Brentjens RJ, Wolchok JD. The future of cancer treatment: immunomodulation, CARs and combination immunotherapy. Nat Rev Clin Oncol 2016;13(05):273-290

14 Tabas I, Glass CK. Anti-inflammatory therapy in chronic disease: challenges and opportunities. Science 2013;339(6116):166-172

15 Graham BS, Ambrosino DM. History of passive antibody administration for prevention and treatment of infectious diseases. Curr Opin HIV AIDS 2015;10(03):129-134

16 Zhou Y, Zong H, Han L, et al. A novel bispecific antibody targeting CD3 and prolactin receptor (PRLR) against PRLR-expression breast cancer. J Exp Clin Cancer Res 2020;39(01):87

17 Han L, Chen J, Ding K, et al. Efficient generation of bispecific IgG antibodies by split intein mediated protein trans-splicing system. Sci Rep 2017;7(01):8360

18 Han L, Zong H, Zhou Y, et al. Naturally split intein Npu DnaE mediated rapid generation of bispecific IgG antibodies. Methods 2019;154:32-37

19 De Groof TWM, Mashayekhi V, Fan TS, et al. Nanobody-targeted photodynamic therapy selectively kills viral GPCR-expressing glioblastoma cells. Mol Pharm 2019;16(07):3145-3156

20 Kijanka M, Dorresteijn B, Oliveira S, van Bergen en Henegouwen PM. Nanobody-based cancer therapy of solid tumors. Nanomedicine (Lond) 2015;10(01):161-174

21 Unciti-Broceta JD, Del Castillo T, Soriano M, Magez S, GarciaSalcedo JA. Novel therapy based on camelid nanobodies. Ther Deliv 2013;4(10):1321-1336

22 Ayyar BV, Arora S, O'Kennedy R. Coming-of-age of antibodies in cancer therapeutics. Trends Pharmacol Sci 2016;37(12):1009-1028

23 Liu XL, Sun TY, Ge QH, et al. Construction of novel bispecific single-domain antibodies (BiSdAbs) with potent antiangiogenic activities. Pharml Fronts 2020;2(01):e64-e76

24 Gao Z, Song C, Li G, et al. Pyrotinib treatment on HER2-positive gastric cancer cells promotes the released exosomes to enhance endothelial cell progression, which can be counteracted by apatinib. OncoTargets Ther 2019;12:2777-2787

25 Vaidyanathan G, McDougald D, Choi J, et al. Preclinical evaluation of ${ }^{18} \mathrm{~F}$-labeled anti-HER2 nanobody conjugates for imaging HER2 receptor expression by immuno-PET. J Nucl Med 2016;57(06):967-973

26 Brenner H, Kloor M, Pox CP. Colorectal cancer. Lancet 2014;383 (9927):1490-1502

27 Houlston RS, Cheadle J, Dobbins SE, et al; COGENT Consortium; CORGI Consortium; COIN Collaborative Group; COINB Collaborative Group. Meta-analysis of three genome-wide association studies identifies susceptibility loci for colorectal cancer at 1q41, 3q26.2, 12q13.13 and 20q13.33. Nat Genet 2010;42(11):973-977

28 Tomlinson IP, Carvajal-Carmona LG, Dobbins SE, et al; COGENT Consortium; CORGI Collaborators; EPICOLON Consortium. Multiple common susceptibility variants near BMP pathway loci GREM1, BMP4, and BMP2 explain part of the missing heritability of colorectal cancer. PLoS Genet 2011;7(06):e1002105

29 Al-Tassan NA, Whiffin N, Hosking FJ, et al. A new GWAS and metaanalysis with 1000Genomes imputation identifies novel risk variants for colorectal cancer. Sci Rep 2015;5:10442

30 Schumacher FR, Schmit SL, Jiao S, et al. Genome-wide association study of colorectal cancer identifies six new susceptibility loci. Nat Commun 2015;6:7138

31 Alameddine RS, Otrock ZK, Awada A, Shamseddine A. Crosstalk between HER2 signaling and angiogenesis in breast cancer: molecular basis, clinical applications and challenges. Curr Opin Oncol 2013;25(03):313-324

32 Yang W, Klos K, Yang Y, Smith TL, Shi D, Yu D. ErbB2 overexpression correlates with increased expression of vascular endothelial growth factors $\mathrm{A}, \mathrm{C}$, and D in human breast carcinoma. Cancer 2002;94(11):2855-2861 
e108 Generating a Novel Bispecific Nanobody to Enhance Antitumor Activity Ge et al.

33 Ishiguro T, Sano Y, Komatsu SI, et al. An anti-glypican 3/CD3 bispecific $\mathrm{T}$ cell-redirecting antibody for treatment of solid tumors. Sci Transl Med 2017;9(410):4291

34 Topp MS, Gökbuget N, Zugmaier G, et al. Phase II trial of the antiCD19 bispecific T cell-engager blinatumomab shows hematologic and molecular remissions in patients with relapsed or refractory B-precursor acute lymphoblastic leukemia. J Clin Oncol 2014;32 (36):4134-4140

35 Yu S, Li A, Liu Q, et al. Recent advances of bispecific antibodies in solid tumors. J Hematol Oncol 2017;10(01):155

36 Zhu JW, ed. Update on Production of Recombinant Therapeutic Protein: Transient Gene Expression. Akron, OH: Smithers Rapra Technology; 2013
37 Ding K, Han L, Zong H, Chen J, Zhang B, Zhu J. Production process reproducibility and product quality consistency of transient gene expression in HEK293 cells with anti-PD1 antibody as the model protein. Appl Microbiol Biotechnol 2017;101(05):1889-1898

38 Zhang X, Han L, Zong H, et al. Enhanced production of anti-PD1 antibody in $\mathrm{CHO}$ cells through transient co-transfection with antiapoptotic genes Bcl- $\mathrm{X}_{\mathrm{L}}$ and Mcl-1. Bioprocess Biosyst Eng 2018;41 (05):633-640

39 Luo M, Zhao M, Cagliero C, et al. A general platform for efficient extracellular expression and purification of Fab from Escherichia coli. Appl Microbiol Biotechnol 2019;103(08):3341-3353

40 Frankel SR, Baeuerle PA. Targeting T cells to tumor cells using bispecific antibodies. Curr Opin Chem Biol 2013;17(03):385-392 